\title{
A LOCATION ALGOLITHM FOR DCS DRIFTING BUOY
}

\author{
Hideo Nishida*
}

\begin{abstract}
A location algorithm for DCS drifting buoy is presented. It is developed as a part of LUT (Local User's Terminal) and designed to be used in a micro-computer. An experiment in which real drifting buoy data were used verified the usefulness of this algorithm.
\end{abstract}

\section{Introduction}

A DCS aboard the satellites in polar orbit can detect the position of a platform. Since the first DCS experiment on NIMBUS-7, this characteristic has been recognized to be quite useful for movable observarion platform. Presently, ARGOS undertakes a function of DCS center and provides sensor and location data to the users in the world.

On the other hand, usefulness of a handy real time receiver terminal which catthes a satelllte down-link transmission wave has become to be recognized gradually. This handy receiver terminal is called LUT (Local User's Terminal) and is useful when we need real time data and a platform location. Japanese Hydrographic Department has been conducting buoy experiment since 1980 aiming at Kuroshio Current fluctuation using ARGOS. The department also initiated a development of LUT in 1982 .

This paper describes an algorithm for locationing of a drifting buoy, which is a part of the LUT system. The algorithm uses basicaly the same principle as NNSS. An outline of NNSS locationing system can be found in many articles (for example, Kimura 1976). The difference between DCS location and NNSS location is that the former uses the Doppler effect of transmission wave from a platform while the latter uses the effect of wave from satellites. The same idea as in the DCS location is studied in SAR (Search and Rescue system) using polar orbit satellites. (Migaki 1981). In this paper, location algorithm especially for öcean observation platform like drifting buoys is discussed and location experiment using real data is given. The computation can be conducted on low cost micro computer. This fact is useful to the users who can not enjoy large computer service.

\section{Algorithm}

(1) Principles

As is explained in every textbook of physics, Doppler shift of frequency depends on the relative speed of transmitter and receiver. In DCS, the transmitter is on a platform and the receiver is onboard DCS itself. The situation is expressed in Fig. 1. The Doppler shift in Fig. 1 situation is given as follows.

$$
\mathrm{F} d=\frac{\mathrm{v}}{\mathrm{c}} \cos \theta \mathrm{F} \quad \quad \cdots \ldots \ldots \ldots \ldots(2,1)
$$


The transmitter which gives a certain value of Doppter shift lies on the cone on whicn $\cos \theta$ in eq. $(2,1)$ is constant. Therefore, the platform should be on the intersection curve of the above cone and sea surface. ARGOS platform transmitts a signal sporadically at every one minutes. When multiple receipts of signal at different satellite positions are obtained, the platforM position can be determined as an intersection point of several intersection curves. It should be noted that there ara two equivalent solutions on both sides of a satellite orbit and we can not determine which the correct one is from the Doppler data only.

Although in practical computation the above theoretical method is not used. Ono of the reason is that precise frequency value is not usually available from a buoy which has drifted for a long time with no maintainance.

(2) Practical Procedure

First we give a rough estimate of a position and frequency and compute the frequency which should be recei-

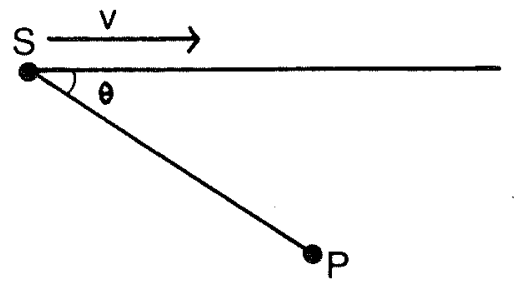

Figure 1 Satellite and platform configuration. $\mathrm{S}$ stands for satellite and $\mathrm{P}$ for platform. The satellite is moving at the velocity of $\mathrm{v}$. ved by DCS. The difference between computed frequency and observed frequency gives an arror which is utilized to compute the first order correction on position and frequency. These corrections are applied to the assumed position and frequency. The new set of position and frequency is put in a same procedure. This convergence process continues to a certain limit where corrections become smaller than the preset values. A flow chart of the procedure is given below.

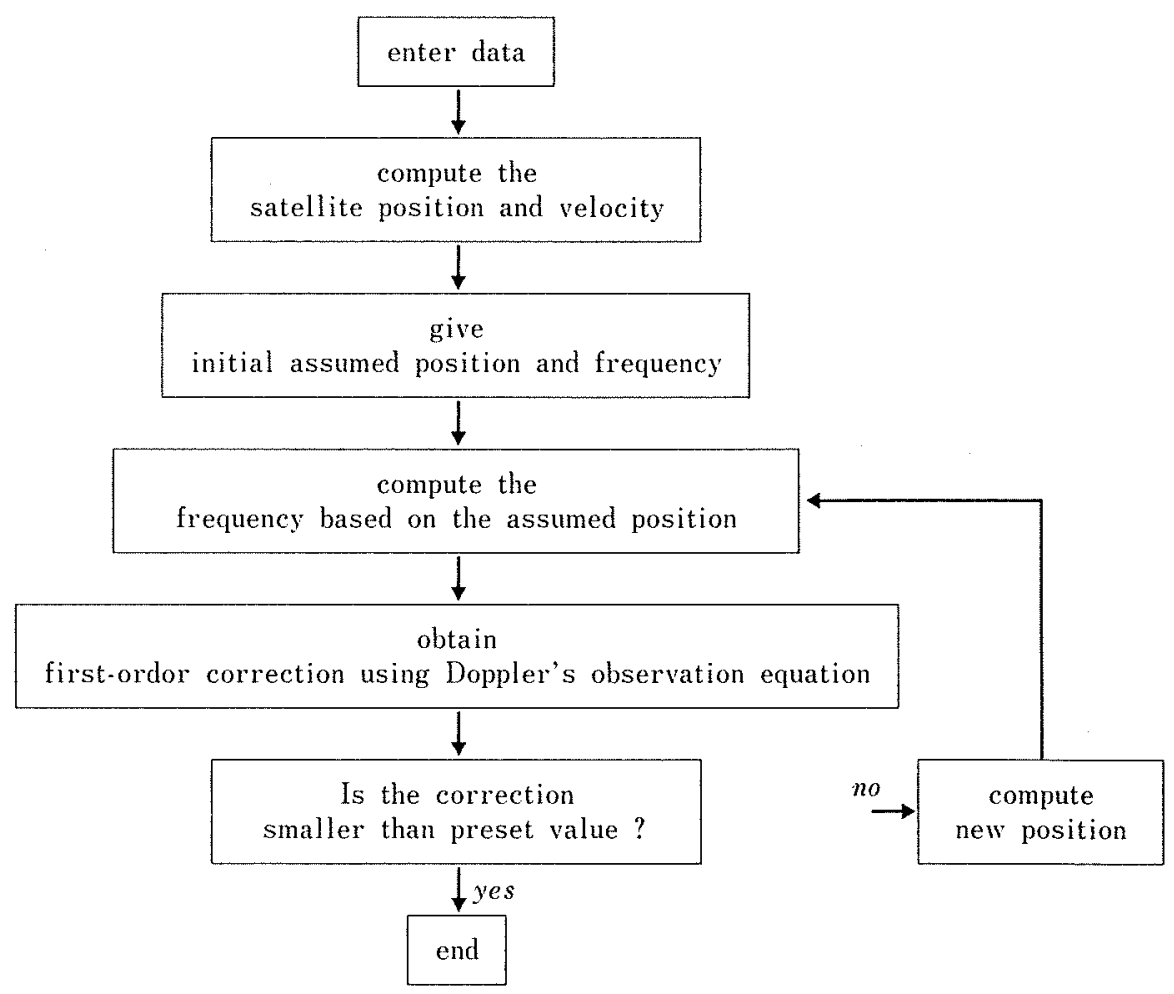




\section{(3) Data}

The time and received frequency values are transmitted from satellites on down-link transmission wave (136.77 and $137.77 \mathrm{MHz}$ ). Usually several set of data are available. Satellite orbit elements, which is used to compute the satellite position and velocity, should be obtained from a certain national satellite center. In Japan JMA (Japan Meteorological Agency) can provide those information.

(4) Satellite Position and Velocity

Satellite orbit is caluculated on the basis of osculating element concept. Six ordital elements with its first-order time derivative are used to give the position and veocity of satellite. Suppose there are $n$ data set (time and frequency) in one satellite pass. We compute the satellite position and velocity at each time point. First we compute the satellite position on orbital plane (Fig. 2). Detailed formulas are given in the Appendix. This set of data is transformed into a geocentric equatorial coordinate.(Fig. 3). Transformation matrix is as follows.

$$
\left(\begin{array}{l}
X \\
Y \\
Z
\end{array}\right)=\left(\begin{array}{ccc}
\cos \Omega & -\sin \Omega & 0 \\
\sin \Omega & \cos \Omega & 0 \\
0 & 0 & 1
\end{array}\right)\left(\begin{array}{ccc}
1 & 0 & 0 \\
0 \cos i & -\sin i \\
0 \sin i & \cos i
\end{array}\right)\left(\begin{array}{ccc}
\cos w & -\sin w & 0 \\
\sin w & \cos w & 0 \\
0 & 0 & 1
\end{array}\right)\left(\begin{array}{l}
x \\
y \\
0
\end{array}\right) \quad \cdots \cdots \cdots \cdots(2 \cdot 2)
$$
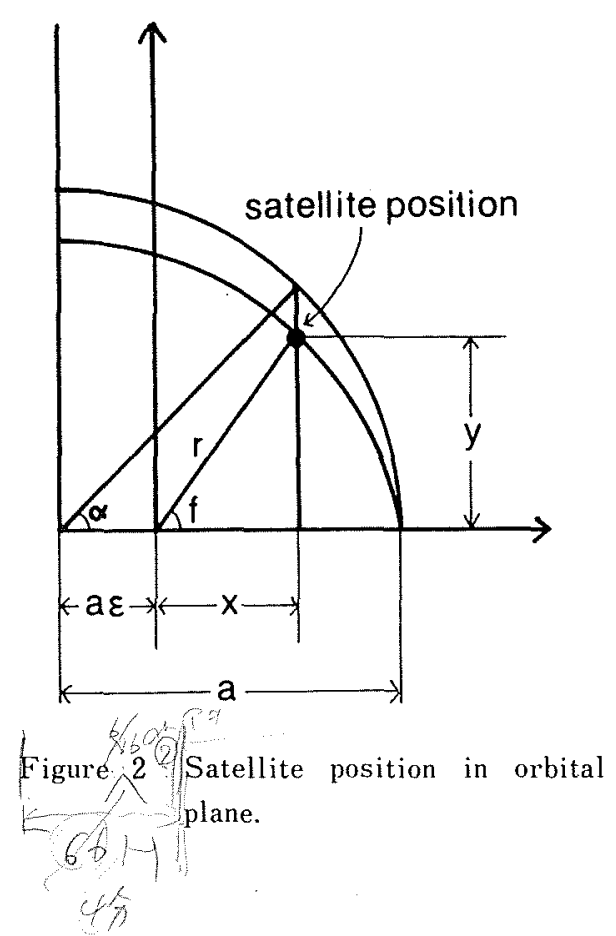

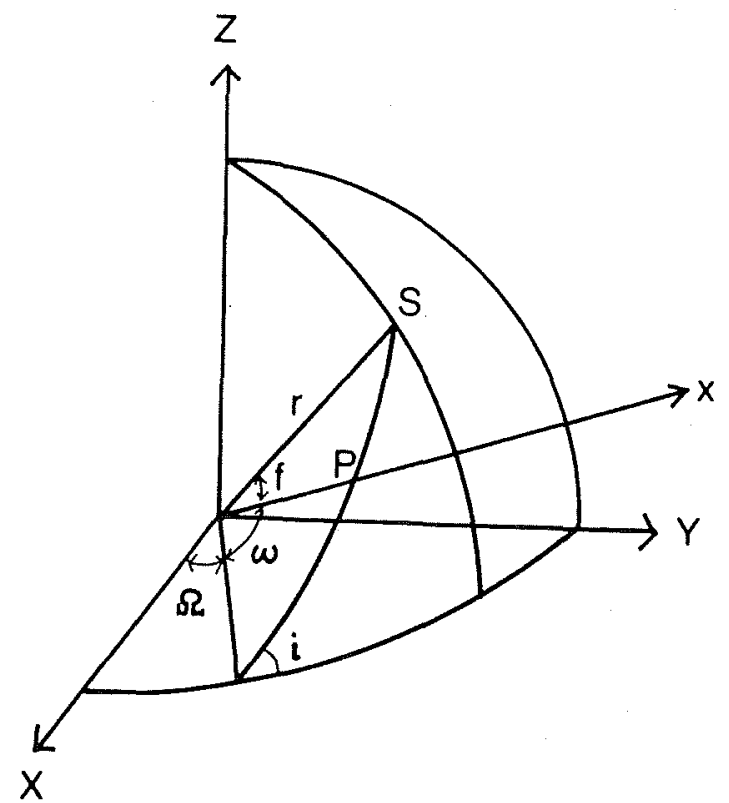

Figure 3 Transformation from satellite orbital plane to equatorial rectangular coordinate. $\mathrm{X}$-axis is directed to vernal equinox, and Z-axis is directed to polaris.

\section{(5) Initial Assumed Position}

There are several ways to give an initial assumed position and frequency. In every day operation former day's position and frequency is usually available. These data are good approximation for true values. When these data are not available, we can roughly estimate the position from frequency difference between succesive receipts (Migaki 1981). Furthermore, we can even devide ocean surface into a mesh, and try every grid point one by one until we a point which produces convergence. 
(6) Doppler's Observation Equation

Doppler shift (Fd) can be expressed with a platform latitude (4), longitude ( $\lambda$ ) and frequency

(F). Opserved frequncy is:

$$
f=\mathrm{F}+\mathrm{Fd}=\mathrm{F}+\mathrm{g}(\phi, \lambda, \mathrm{F})
$$

The difference between observed frequency and calculated frequency based on assumed position can be expanded with the differences of latitude $(\Delta \varphi)$, longitude $(\Delta \lambda)$ and frequency $(\Delta F)$ as :

$$
\Delta f=\frac{\mathrm{d} f}{\mathrm{~d} \psi} \Delta \psi+\frac{\mathrm{d} f}{\mathrm{~d} \lambda} \Delta \lambda+\frac{\mathrm{d} f}{\mathrm{~d} F} \Delta \mathrm{F}=\frac{\mathrm{d} g}{\mathrm{~d} \phi} \Delta \psi+\frac{\mathrm{d} g}{\mathrm{~d} \phi} \Delta \lambda+\left(\mathrm{H} \frac{\mathrm{d} g}{\mathrm{~d} F}\right) \Delta \mathrm{F} \cdots \cdots \cdots \cdots \cdots \cdot(2 \cdot 4)
$$

Considering $(2 \cdot 1), \frac{\mathrm{dg}}{\mathrm{d} \varphi}, \frac{\mathrm{dg}}{\mathrm{d} \lambda}, \frac{\mathrm{dg}}{\mathrm{dF}}$ can be expressed as :

$$
\left.\begin{array}{l}
\frac{\mathrm{d} g}{\mathrm{~d} \psi}=\frac{\nu \mathrm{F}}{c} \frac{\mathrm{d}(\cos \theta)}{\mathrm{d} \psi} \\
\frac{\mathrm{d} g}{\mathrm{~d} \lambda}=\frac{\nu \mathrm{F}}{c} \frac{\mathrm{d}(\cos \theta)}{\mathrm{d} \lambda} \\
\frac{\mathrm{d} g}{\mathrm{dF}}=\frac{\nu}{c} \cos \theta
\end{array}\right\}
$$

In order to execute the differentiation in $(2 \cdot 5)$, we must have $\cos \theta$ expressed aparently with $\varphi, \lambda$ $\lambda, \mathrm{F})$. In equatorial rectangular coordinate, $\cos \theta$ can be expressed as :

$$
\cos \theta=\frac{\frac{d X_{s}}{d t}\left(X_{P}-X_{S}\right)+\frac{d Y_{S}}{d t}\left(Y_{P}-Y_{S}\right)+\frac{d X_{S}}{d t}\left(Z_{P}-Z_{s}\right)}{\sqrt{\left(\frac{d X_{s}}{d t}\right)^{2}+\left(\frac{d Y_{s}}{d t}\right)^{2}+\left(\frac{d Z_{S}}{d t}\right)^{2}} \sqrt{\left(X_{P}-X_{s}\right)^{2}+\left(Y_{P}-Y_{S}\right)^{2}+\left(Z_{P}-X_{s}\right)^{2}}} \cdots \cdots(2 \cdot 6)
$$

where $\left(X_{s}, Y_{s}, Z_{s}\right)$ is a satellite position, $\left(\frac{d X_{s}}{d t}, \frac{d Y_{s}}{d t}, \frac{d Z_{s}}{d t}\right)$ is a satellite velocity and $\left(X_{p}, Y_{p}\right.$, $Z_{p}$ ) is a platform position. Transformation from $(\varphi, \lambda)$ to epuatorial rectangular coordiate can be made using the equation (Tsubokawa 1974),

$$
\left(\begin{array}{l}
X_{P} \\
Y_{P} \\
X_{P}
\end{array}\right)=\left(\begin{array}{l}
(N+h g) \cos \psi \cos \left(\lambda_{0}+\omega t+\lambda\right) \\
(N+h g) \cos \psi \cos \psi \sin \left(\lambda_{0}+\omega t+\lambda\right) \\
\left\{\left(1-e^{2}\right) N+h g\right\} \sin \psi
\end{array}\right)
$$

where $\quad \mathrm{N}=\mathrm{a}\left(1-\mathrm{e}^{2}\right) \sin ^{2} \varphi$

where a is semi major-axis of the reference ellipsoid

e is eccentricity of the reference ellipsoid

hg is geoid height from reference ellipsoid

$\omega$ is angular velocity of the Earth

and $\lambda_{0}$ is Greenich sidereal time

Constants for reference ellipsoid WGS-72 are given in Appendix.

With the use of $(2 \cdot 6)$ and $(2 \cdot 7)$, the differeniaton in $(2 \cdot 5)$ can be made. Complete expression is given in Appendix. We conduct a computation at each receiving time, and obtain a set of equations in the form of $(2 \cdot 4)$. Usually the number of equations is greater than three, that is, the number 
of unknown variables. In that case, we apply least square method to the set of equations to obtain correction values $(\Delta \varphi, \Delta \lambda, \Delta \mathrm{F})$. With the use of these correction values, we have a new set of position and frequency. As is explained in $2 \cdot 2$, this procedure repeats until we have sufficiently precise values.

\section{Experiment}

An experiment was made using data taken in Oct. 1, 1981. The platform was a drifting buoy (ID, No. 2029) launched by the Hydrographic Department. A satellite pass and the receiving points are shown in Fig. 4, Five data were above noise level. After some trial and error, the computation converged and a platform position $\left(24.81^{\circ} \mathrm{N}, 141.70^{\circ} \mathrm{E}\right)$ and frequency $(401650216 \mathrm{~Hz})$ were obtained. This position is shown as a solid triangle in Fig. 4. In order to investigate the effect of a choice ofan initial assumed position on the convergence, every one-degree grid point in the area enclosed by $35^{\circ}$ and $5^{\circ}$ latitude and $125^{\circ}$ and $163^{\circ}$ longitude were tested as an initial assumed position.

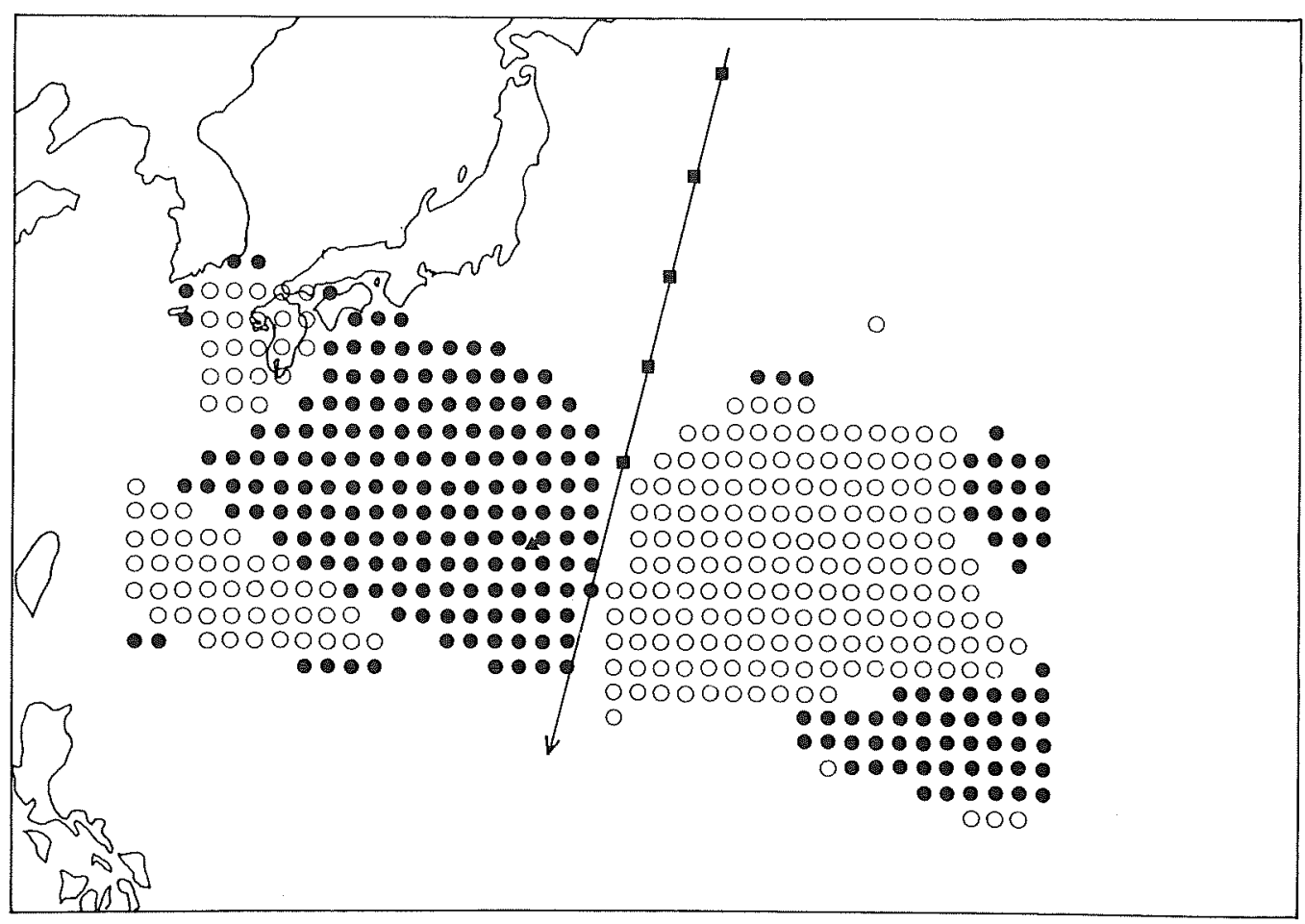
: Location of satellite
A : Caluculated position
- Initial positions which went to the correct one
$O$ : Initial positions which went to the mirror image

Figure 4 Map showing the dependence of choice of initial-position upon the convergence computation. For details see the text. 
As an initial frequency, the true value that is, $401650216 \mathrm{~Hz}$ adopted. In this computation final convergence is judged when correction values (of latitude and longitude) became less than $10^{-5} \mathrm{rad}$. Anb also, when the number of repetition went over twenty, the computation was judged as divergence. Solid circles in Fig. 4 show points from where computation converged into the true point. Open circles show points from where the computation converged into the mirror point. As is shown in Fig. 4, a very large area can be taken as an initial assumed position. All the time when convergence was reached, the number of repetition was less than ten. Using a personal computer (NEC model PC-8800), a convergence computation was accomplised in less than two minutes.

\section{Appendix 1}

Satellite position in orbital plane is obtained in the following way. The position $(X, Y)$ in

Fig. 2 is expressed with semi-major axis (a), eccentric anomaly $(\alpha)$ and eccentricity $(\varepsilon)$ as :

$$
\left(\begin{array}{l}
X \\
Y
\end{array}\right)=\left(\begin{array}{ll}
r & \cos f \\
r & \sin f
\end{array}\right)=\left(\begin{array}{l}
\mathrm{a}(\cos \alpha-\varepsilon) \\
\mathrm{a} \sqrt{1-\varepsilon^{2} \sin \alpha}
\end{array}\right)
$$

We have a relation between eccentric anomaly $(\alpha)$ and mean anomaly (M) as:

$$
\mathrm{M}=\alpha-\varepsilon \sin \alpha
$$

Mean anomaly (M) can be expressed as a function of time as :

$$
M=\frac{2 \pi}{T}\left(t-t_{P}\right)
$$

Where $\mathrm{T}$ is period and tp is time when crossing perigee. First we caluculate mean anomaly using $(A \cdot 3)$, then we caluculate eccentric anomaly $(\alpha)$ using $(A \cdot 2)$. In solving $(A \cdot 2)$, we use iterative method because $(A \cdot 2)$ is a transcendental equation. When we have eccentric anomaly $(\alpha)$, the position can be easily obtained using $(A \cdot 1)$.

Satellite velocity in orbital plane can be obtained as follows. Differentiation of $(A \cdot 1)$ with time gives us,

$$
\left(\begin{array}{l}
\frac{d X}{d t} \\
\frac{d y}{d Y}
\end{array}\right)=\left(\begin{array}{l}
-a \sin \alpha \frac{d \alpha}{d t} \\
a\left(1-\varepsilon^{2}\right) \frac{1}{2} \cos \alpha \frac{d \alpha}{d t}
\end{array}\right)
$$

Differentiating $(A \cdot 2)$ with time, we derive after some manipulation,

$$
\frac{\mathrm{d} \alpha}{\mathrm{dt}}=\frac{\frac{\mathrm{dM}}{\mathrm{dt}}}{1-\varepsilon \cos \alpha}
$$

$\frac{d M}{d t}$ can be easily obtained using $(A \cdot 3)$. With the use of $(A \cdot 5)$, we can have a velocity in a orbital plane $\left(\frac{d X}{d t}, \frac{d Y}{d t}\right)$. Those two data $(X \cdot Y),\left(\frac{d X}{d t}, \frac{d Y}{d t}\right)$ can be transformed into the equatorial rectangular coordinates using $(2 \cdot 2)$. 


\section{Appendix 2}

Apparent form of $\frac{\mathrm{d}(\cos \theta)}{\mathrm{d} \varphi}$ and $\frac{\mathrm{d}(\cos \theta)}{\mathrm{d} \lambda}$ in $(2 \cdot 5)$ are obtained as follows. We differentiate equation $(2 \cdot 6)$ with $\varphi$ and $\lambda$. Because satellite position $\left(X_{s}, Y_{s}, Z_{s}\right)$ and satellite velocity $\left(\frac{d X_{s}}{d t}\right.$, $\left.\frac{d Y_{s}}{d t}, \frac{d Z_{s}}{d t}\right)$ are both indepentdent of $(\varphi, \lambda)$, the differentiation gives us for,

$$
\begin{aligned}
& \frac{\mathrm{d}(\cos \theta)}{\mathrm{d} \phi}=\frac{1}{\sqrt{\left(\frac{\mathrm{dX}}{\mathrm{dt}}\right)^{2}+\left(\frac{\mathrm{d} \mathrm{Y}_{\mathrm{s}}}{\mathrm{dt}}\right)^{2}+\left(\frac{\mathrm{d} \mathrm{Z}_{\mathrm{s}}}{\mathrm{dt}}\right)^{2}}} \times \mathrm{X}_{d}^{2}+\mathrm{Y}_{d}^{2}+\mathrm{Z}_{d}^{2} \frac{\mathrm{A}^{*}}{\mathrm{X}_{d}^{2}+\mathrm{Y}_{d}^{2}+\mathrm{Z}_{d}^{2}} \\
& \mathrm{~A}^{*}=\left(\frac{\mathrm{d} \mathrm{X}_{\mathrm{s}}}{\mathrm{dt}} \frac{\mathrm{dXd}}{\mathrm{d} \phi}+\frac{\mathrm{d} \mathrm{Y}_{\mathrm{s}}}{\mathrm{dt}} \frac{\mathrm{d} \mathrm{Y}_{d}}{\mathrm{~d} \phi}+\frac{\mathrm{d} \mathrm{Z}_{\mathrm{s}}}{\mathrm{dt}} \frac{\mathrm{d} \mathrm{Z}_{d}}{\mathrm{~d} \phi}\right) \sqrt{\mathrm{X}_{d}^{2}+\mathrm{Y}_{d}^{2}+\mathrm{Z}_{d}^{2}} \\
& -\left(\frac{\mathrm{dX} \mathrm{X}_{\mathrm{s}}}{\mathrm{dt}} \mathrm{X}_{d}+\frac{\mathrm{d} \mathrm{Y}_{\mathrm{s}}}{\mathrm{dt}} \mathrm{Y}_{d}+\frac{\mathrm{d} \mathrm{Z}_{\mathrm{s}}}{\mathrm{dt}} \mathrm{Z}_{d}\right) \frac{1}{2}\left(\mathrm{X}_{d}^{2}+\mathrm{Y}_{d}^{2}+\mathrm{Z}_{d}^{2}\right)^{-\frac{1}{2}} \frac{\alpha}{\alpha \psi}\left(\mathrm{X}_{d}^{2}+\mathrm{Y}_{d}^{2}+\mathrm{Z}_{d}^{2}\right)
\end{aligned}
$$

where

$$
\left(\begin{array}{l}
\mathrm{X}_{d} \\
\mathrm{Y}_{d} \\
\mathrm{Z}_{d}
\end{array}\right)=\left(\begin{array}{l}
\mathrm{X}_{\mathrm{P}}-\mathrm{X}_{\mathrm{S}} \\
\mathrm{Y}_{\mathrm{P}}-\mathrm{Y}_{\mathrm{S}} \\
\mathrm{X}_{\mathrm{P}}-\mathrm{Z}_{\mathrm{S}}
\end{array}\right)
$$

We have completely the same equation as $(A \cdot 6)$ for $\lambda$ except $\varphi$ should be $\lambda$. replaced by $\lambda$. Upon inspection of equation $(A \cdot 6)$, we know that the terms which we need are $\frac{d X_{d}}{d}, \frac{d Y_{d}}{d \varphi}, \frac{d Z_{d}}{d \varphi}$. The situation is the same for $\lambda$-terms, and finally we arr to caluculate six terms, i.e, $\frac{d X_{d}}{d \varphi}, \frac{d X_{d}}{d \varphi}, \frac{d Z_{d}}{d \varphi}$, $\frac{d X_{d}}{d \lambda}, \frac{d Y_{d}}{d \lambda}, \frac{d Z_{d}}{d \lambda}$.

The caluculation can be made using $(2 \cdot 7)$,

$$
\begin{aligned}
\left(\begin{array}{c}
\frac{\mathrm{d} \mathrm{X}_{d}}{\mathrm{~d} \psi} \\
\frac{\mathrm{d} \mathrm{Y}_{d}}{\mathrm{~d} \psi} \\
\frac{\mathrm{d} \mathrm{Z}_{d}}{\mathrm{~d} \psi}
\end{array}\right)=\left(\begin{array}{c}
\frac{\mathrm{d} \mathrm{X}_{\mathrm{P}}}{\mathrm{d} \psi} \\
\frac{\mathrm{d} \mathrm{Y}_{\mathrm{p}}}{\mathrm{d} \psi} \\
\frac{\mathrm{d} \mathrm{Z}_{\mathrm{P}}}{\mathrm{d} \psi}
\end{array}\right) \\
=\left(\begin{array}{l}
\frac{\mathrm{dN}}{\mathrm{d} \psi} \cos \psi \cos \left(\lambda_{0}+\omega \mathrm{t}+\lambda\right)-(\mathrm{N}+\mathrm{hg}) \sin \psi \cos \left(\lambda_{0}+\omega \mathrm{t}+\lambda\right) \\
\frac{\mathrm{dN}}{\mathrm{d} \psi} \cos \psi \sin \left(\lambda_{0}+\omega \mathrm{t}+\lambda\right)-(\mathrm{N}+\mathrm{hg}) \sin \psi \sin \left(\lambda_{0}+\omega \mathrm{t}+\lambda\right) \\
\left(1-\mathrm{e}^{2}\right) \frac{\mathrm{dN}}{\mathrm{d} \psi} \sin \psi+1\left(1-\mathrm{e}^{2}\right) \mathrm{N}+\mathrm{hg} \cos \psi
\end{array}\right) \\
\left(\begin{array}{l}
\frac{\mathrm{d} \mathrm{X}_{d}}{\mathrm{~d} \lambda} \\
\frac{\mathrm{d} \mathrm{Y}_{d}}{\mathrm{~d} \lambda} \\
\frac{\mathrm{d} \mathrm{Z}_{d}}{\mathrm{~d} \lambda}
\end{array}\right)=\left(\begin{array}{l}
\frac{\mathrm{d} \mathrm{X}_{\mathrm{P}}}{\mathrm{d} \lambda} \\
\frac{\mathrm{d} \mathrm{Y}_{\mathrm{P}}}{\mathrm{d} \lambda} \\
\frac{\mathrm{d} \mathrm{P}_{\mathrm{P}}}{\mathrm{d} \lambda}
\end{array}\right)=\left(\begin{array}{l}
-(\mathrm{N}+\mathrm{hg}) \cos \psi \sin \left(\lambda_{\mathrm{o}}+\omega \mathrm{t}+\lambda\right) \\
(\mathrm{N}+\mathrm{h}) \cos \psi \cos \left(\lambda_{\mathrm{o}}+\omega \mathrm{t}+\lambda\right)
\end{array}\right)
\end{aligned}
$$

where

$$
\frac{\mathrm{d} N}{\mathrm{~d} \psi}=\mathrm{a}\left(1-\mathrm{e}^{2} \sin ^{2} \psi\right)^{-\frac{3}{2}} \mathrm{e}^{2} \sin \psi \cos \psi
$$




\section{Appendix 3}

Least square method as applied to $(2 \cdot 4)$ is the following. Equations in the form of $(2 \cdot 4)$ derived for different time points form simultaneous liner equations for the unknown variables. ( $\Delta \varphi, \Delta$ $\lambda, \Delta \mathrm{F}$,$) .$

$$
\begin{array}{r}
\Delta f_{k}=\left(\frac{\mathrm{dg}}{\mathrm{d} \psi}\right)_{k} \Delta \psi+\left(\frac{\mathrm{dg}}{\mathrm{d} \phi}\right)_{k} \Delta \lambda+\left(1+\frac{\mathrm{dg}}{\mathrm{d} \mathrm{F}}\right)_{k} \Delta f \\
(\mathrm{~K}=1, \cdots \cdots \cdots \cdots \cdots, \mathrm{n})
\end{array}
$$

When the number $\mathrm{n}$ is greater than three, we apply least square method. Normal equations for (A .

11) are :

$$
\left(\begin{array}{l}
\Sigma \mathrm{a}_{k} \Delta f_{k} \\
\Sigma \mathrm{b}_{k} \Delta f_{k} \\
\Sigma \mathrm{c}_{k} \Delta f_{k}
\end{array}\right)=\left(\begin{array}{ccccc}
\Sigma \mathrm{a}_{k} \mathrm{a}_{k} & \sum \mathrm{a}_{k} \mathrm{~b}_{k} & \sum \mathrm{a}_{k} \mathrm{c}_{k} \\
\Sigma \mathrm{b}_{k} & \mathrm{a}_{k} & \Sigma \mathrm{b}_{k} \mathrm{~b}_{k} & \sum \mathrm{b}_{k} \mathrm{c}_{k} \\
\Sigma \mathrm{c}_{k} & \mathrm{a}_{k} & \Sigma \mathrm{c}_{k} \mathrm{~b}_{k} & \sum \mathrm{c}_{k} \mathrm{c}_{k}
\end{array}\right)\left(\begin{array}{c}
\Delta \psi \\
\Delta \lambda \\
\Delta \mathrm{F}
\end{array}\right)
$$

where

$$
\mathrm{a}_{k}=\left(\frac{\mathrm{dg}}{\mathrm{d} \psi}\right)_{k}, \quad \mathrm{~b}_{k}=\left(\frac{\mathrm{dg}}{\mathrm{d} \lambda}\right)_{k}, \quad \mathrm{c}_{k}=\left(1+\frac{\mathrm{dg}}{\mathrm{dF}}\right)_{k}
$$

\section{Appendix 4}

Constants of the reference ellipsoid WGS-72 are as follows.

$$
\begin{aligned}
& \mathrm{a}=6378135.00 \text { (meter) } \\
& \mathrm{f}(\text { flattening coeff. })=1 / 298.2600
\end{aligned}
$$

Eccentricity (e) can be obtained from $f$ using the relation :

$$
\mathrm{e}^{2}=(2-\mathrm{f}) \mathrm{f}
$$

\section{References}

Kimura, K. 1977 : satellite Navigation (in Japanese), Kaibundo, Tokyo, pp190.

Migaki, M. 1981 : On a method of position location for a Search and Rescue System with an orbiting satellite, Electoronic Navigation Res. Inst. Pap., No. 34, pp. 37-60.

Mueller, I.I. 1964 : Introduction to Satellite Geodesy, Frederic Ungar Publishing Co., New York, pp. 415.

Tsubokawa, I. 1974 : Outline of Geodesy (in Japanese), Japan Geodetic Society, Tokyo, pp. 511.

\section{漂流ブイの位㯰算出アルゴリズム（要旨） \\ 西田英男}

DCSを利用した漂流ブイの位置算出アルゴリズムを作成した。これはLUTの一部として開発したもので， マイクロコンピューターで使用できるようになっている．実際の漂流ブイデータを利用して計算を行った結 果，満足できる精度が得られた。 